\title{
COMPETENCIAS Y DESAFÍOS DEL DIRCOM EN UN PAÍS EN DESARROLLO
}

\author{
Baquerizo Neira, Gabriela ${ }^{1}$ \\ Universidad Casa Grande, Ecuador \\ gbaquerizo@casagrande.edu.ec
}

\author{
Correa Vélez, Karen ${ }^{2}$ \\ Universidad Casa Grande, Ecuador \\ kcorrea@casagrande.edu.ec
}

Yaguache, Jenny ${ }^{3}$

Universidad Técnica Particular de Loja, Ecuador

ïyaguache@utpl.edu.ec

Material original autorizado para su primera publicación en la revista

académica REDMARKA. Revista Digital de Marketing Aplicado.

https://doi.org/10.17979/redma.2018.01.021.4839

Recibido: 11 enero 2018

Aceptado: 8 agosto 2018

\footnotetext{
1 Doctora en Comunicación Social por la Universidad Austral, Argentina. Magíster en Dirección de Comunicación y Nuevas Tecnologías. Publicaciones en IEEE Transactions on Emerging Topics in Computing. Áreas de investigación: reputación, liderazgo político femenino, comunicación estratégica, redes sociales y sentiment analysis.

2 Magíster en Comunicación Organizacional, periodista económica por más de 20 años y docente de la Facultad Mónica Herrera de la Universidad Casa Grande de Guayaquil, donde dirige el proyecto de investigación Semillero: Diagnóstico de Comunicación de Crisis: Caso ecuatoriano (2017-2018). Profesora de la Universidad de Especialidades Espíritu Santo para las materias de Periodismo y Comunicación Organizacional.

${ }^{3}$ Doctora en Ciencias de la Comunicación por la Universidad de Santiago de Compostela, España y profesora de las escuelas de Comunicación Social y Relaciones Públicas de la Universidad Técnica Particular de Loja. Coordinadora del Grupo de Investigación Gestión de la Comunicación Estratégica. Autora de diversos artículos y textos académicos.
}

REDMARKA IMARKA-Universidad de A Coruña - CIECID

Año XI, Número 21, (2018), vol. 1 pp. 53-77

http://www.redmarka.net/ ISSN 1852-2300 


\section{Resumen}

La investigación revela que en Ecuador los gestores de comunicación de las cincuenta empresas analizadas cuentan con un mínimo de 5 años de experiencia en la gestión de comunicación y un $18 \%$ del total de casos estudiados tienen entre 6 y 14 años de experiencia. La edad de los gestores de comunicación fluctúa entre los 25 y 39 años en un mayor porcentaje.

En esta investigación se procura una aproximación al perfil actual del Dircom en el Ecuador para identificar sus habilidades y reconocer cómo se adapta a las condiciones propias de cada sector. Para ello, se seleccionaron 50 corporaciones de diversos sectores productivos y de servicios del país, que tienen al menos 10 años en el mercado, tiempo que coincide con periodo presidencial ininterrumpido más extenso que ha existido en el Ecuador y que en los últimos años ha transitado por un proceso de desaceleración económica.

En esta la investigación se evidenció que, a pesar de una incipiente oferta de programas de formación en dirección de comunicación los profesionales que actualmente ocupan los cargos directivos en los departamentos de Comunicación Institucional de las empresas del país acceden a programas de formación de cuarto y tercer nivel, a fin de adquirir los conocimientos y habilidades para solventar las demandas comunicacionales del mercado. Esta perspectiva les permite ubicarse en niveles estratégicos de la organización, y cada día menos operativos, según los datos obtenidos; sin embargo, también hay responsables de comunicación con una titulación diferente a la comunicación estratégica.

Los resultados del estudio también revelaron que los Dircom seleccionados dedican su gestión a la comunicación integral y que esta, en ocasiones, se desarrolla con el apoyo de agencias. Ejecutan además comunicación interna en la 
medida de las posibilidades, pues esta acción importante para la organización es coordinada con el departamento de recursos humanos.

Palabras clave: Dircom, comunicación organizacional, estrategia, empresa, Ecuador

\section{Abstract}

The research reveals that in Ecuador the communication managers of the fifty companies analyzed have a minimum of 5 years of experience in communication management and $18 \%$ of the total cases studied have between 6 and 14 years of experience. The age of communication managers fluctuates between 25 - 39 years in a greater percentage.

This research seeks an approximation to the current profile of Dircom in Ecuador to identify their skills and recognize how they adapts to the conditions of each sector. To this end, 50 corporations from different productive and service sectors of the country were selected, which have been in the market for at least 10 years, a time that coincides with the longest uninterrupted presidential period that has existed in Ecuador. Transited by a process of economic slowdown.

In this research it was evidenced that, in spite of an incipient offer of training programs in communication management, the professionals currently occupying the management positions in the Institutional Communication departments of the companies of the country have access to postgraduate programs, in order to acquire the knowledge and skills to solve the market's communication demands. This perspective allows them to be located at strategic levels of the organization, and each day less operational, according to the data obtained. However, there are also communication managers with a different degree than strategic communication.

The results of the study also revealed that the Dircom selected devote their management to the integral communication and that this, sometimes, is developed

REDMARKA IMARKA-Universidad de A Coruña - CIECID 
with the support of agencies. They also execute internal communication to the extent of the possibilities, because this important action for the organization is coordinated with the human resources department.

Keywords: Dircom, organizational communication, strategy, company, Ecuador

\section{ROL DEL COMUNICADOR EN UN CONTEXTO GLOBALIZADO}

Si bien, en la década de los noventa, en España el profesional de la comunicación ya se configura como Dircom, en América Latina este fenómeno es reciente y su rol se ha visto impactado por la vertiginosa transformación del ecosistema mediático. Hoy, la sociedad digital demanda un perfil "estratega generalista polivalente" del comunicador, experto en el manejo de las redes sociales y la comunicación móvil e inmerso en una cultura permeada por la comunicación digital interactiva (Joan Costa, 2010:194).

Esta reconfiguración social ha generado cambios en la comunicación corporativa, que pasó de ser una "tarea técnica a ser un área estratégica" (Dircom, 2015:12). Esto, ha provocado la transformación conceptual del diseño y estructura de las organizaciones, que se han visto abocadas a trasladar su naturaleza de orden mecanicista a una de tipo orgánica (Burns \& Stalker, 1961). En este sentido, el fenómeno Dircom surge como un profesional con una mirada holística de los procesos y flujos de la comunicación en las organizaciones, un experto en la gestión de la identidad y la cultura que procura trabajar en red y mantener una relación con los públicos internos y externos de la organización. En definitiva, un gestor estratégico de los intangibles, es decir, de la reputación corporativa (Dircom, 2015; Villafañe, 1999).

Esta evolución del perfil del director de comunicación se caracteriza por tener una mirada omnicomprensiva de la organización, que más allá de dar importancia a los réditos económicos se preocupa por la gestión de sus intangibles, los valores y la reputación de la empresa (Mut, 2013). Además, posee un componente del

REDMARKA IMARKA-Universidad de A Coruña - CIECID 
management, esto hace que su rol en la organización esté "más alineado a los negocios y cada vez más alejado de la comunicación clásica" (Michael Ritter, 2011:23). Así, se lo reconoce como un especialista capaz de realizar diagnósticos de los flujos y planes comunicacionales, identificar problemas y proponer e implementar planes de acción para mejorar la comunicación hacia dentro o fuera de la empresa (Pérez, 2012).

\section{CONTEXTO ECUATORIANO DEL DIRCOM}

Una investigación reciente realizada por el Observatorio de la Comunicación Estratégica en Ecuador ${ }^{4}$ identificó que en la mayoría de organizaciones la gestión de la comunicación es departamental y está centrada en actividades como: "relaciones públicas, marketing y publicidad, y comunicación digital"; incluso, en ocasiones las empresas contratan a terceros (i.e. agencias de publicidad, relaciones públicas y comunicación; empresas de investigación de mercado, entre otras) para esta tarea (Fanny Paladines Galarza, Jenny Yaguache Quichimbo \& Verónica Altamirano Benítez, 2015:15). Esto confirma que en las organizaciones ecuatorianas el manejo de la comunicación no es sistémico, está fragmentado, lo que ocasiona un impacto en la imagen, identidad, discurso e incluso en el presupuesto destinado para la comunicación.

En función de lo expuesto, en esta investigación a partir del análisis de entrevistas en profundidad a 50 expertos y directivos de organizaciones públicas y privadas de los sectores productivo y de servicios, se determina cuáles son las habilidades que actualmente demanda el mercado ecuatoriano a los profesionales de la comunicación en un entorno cambiante afectado por la desaceleración económica.

\footnotetext{
4 "Primer proyecto formal realizado desde la academia en Ecuador y en contraste con la realidad de otros países, pretende ser el repositorio donde los profesionales, directores, estudiantes y docentes de la comunicación estratégica encuentren resultados y temas de interés para fortalecer sus trabajos". Recuperado el 05 de marzo de 2018 de https://observatoriocom.utpl.edu.ec/.
}

REDMARKA IMARKA-Universidad de A Coruña - CIECID

Año XI, Número 21, (2018), vol. 1 pp. 53-77

http://www.redmarka.net/ ISSN 1852-2300 


\section{METODOLOGÍA}

La presente investigación indaga en la formación académica, profesional y experimental de los gestores de comunicación de 50 empresas del Ecuador. Las entrevistas se realizaron durante los meses de agosto y octubre de 2017. La intención de la investigación es aproximarse al perfil actual del Dircom en el Ecuador y cómo se adapta a las condiciones propias de cada sector ${ }^{5}$.

Para la muestra seleccionada se consideró también a agencias de comunicación y de estudios de mercado que en muchos casos compensaron la carencia de estos departamentos en las empresas ecuatorianas y que nacieron como emprendimientos de profesionales que se especializaron trabajando para multinacionales ya sea en relación de dependencia o a través de asesorías.

"En mi caso puntual, tengo formación universitaria de comunicador social con especialización en redacción creativa y publicitaria, y durante la gran mayoría de mis 17 años de carrera he ejercido como comunicador corporativo" (Piedra, L., entrevista personal)

Se emplea la entrevista como herramienta de investigación porque la particularidad de ser de carácter abierto permite al investigador, a través de un trabajo reflexivo, relacionar conceptos e incorporar experiencias de campo durante el análisis e interpretación de los resultados (Hernández Carrera, 2014). La entrevista de tipo semiestructurada se compone de tres apartados, a saber: gestión de la comunicación, relación con los stakeholders y comunicación de crisis. La guía de preguntas se elaboró considerando el perfil del Chief Communication Officer ( $C C O$ ), profesional de la comunicación qué de relacionarse exclusivamente con los medios, se convierte en gestor de la reputación de la

\footnotetext{
${ }^{5}$ Por ejemplo, en el caso de las empresas del sector de la construcción por ser proveedoras de obra pública, delegan la responsabilidad de relacionarse con los medios de comunicación y con otros públicos a las instituciones estatales o gobiernos seccionales que las contratan.
}

REDMARKA IMARKA-Universidad de A Coruña - CIECID

Año XI, Número 21, (2018), vol. 1 pp. 53-77

http://www.redmarka.net/ ISSN 1852-2300 
empresa con campo de acción en áreas como: "comunicación corporativa, imagen de marca, responsabilidad social corporativa, comunicación externa e interna, plataformas digitales y redes sociales, marketing y publicidad" (Burson Marsteller, 2013:8).

A través de la entrevista semiestructurada se procuró indagar en cuestiones como: perfil académico y profesional del gestor de comunicación de la empresa seleccionada, a cargo de qué tipo de actividades está y si estas corresponden a roles estratégicos u operativos. Además, ¿qué es para ellos la comunicación estratégica? ¿conocer si el departamento que dirigen se encarga de gestionar la comunicación interna y externa de la organización o si contratan algún proveedor?

La selección de los 50 casos de estudio se hizo considerando el ranking empresarial Top 1000 de Revista EKOS $(2015)^{6}$ y el listado sectorial de las 500 mayores empresas del Ecuador de Revista Vistazo (2016) ${ }^{7}$. A partir de estas bases se identificaron las organizaciones considerando lo siguiente: por una parte, se excluyen los ministerios debido al inicio del nuevo periodo de administración gubernamental (2017-2021), pero se mantienen las empresas estatales de los sectores estratégicos debido a la transformación tecnológica de la gestión de la comunicación en la administración pública durante el gobierno de la Revolución Ciudadana (2007-2017); también se descartan las Organizaciones No Gubernamentales (ONG) porque no corresponden a la categoría empresarial y por ende no cumplen con el mínimo de 30 empleados en relación de dependencia y en muchas ocasiones el personal es flotante.

Por otra parte, se mantienen las instituciones públicas y privadas de Educación Superior que ostentan la categoría A o B como producto del proceso de evaluación, acreditación y re-categorización institucional realizado por el Ceaaces

\footnotetext{
${ }^{6}$ Para ampliar la información revisar

http://www.ekosnegocios.com/negocios/verArticuloContenido.aspx ?idArt=6259

${ }^{7}$ Para ampliar la información revisar http://www.vistazo.com/seccion/proyectos-especiales/500empresas
} 
desde el año $2009^{8}$. También se consideran, las empresas aseguradoras, las organizaciones del sector de servicios básicos y las instituciones de carácter político (i.e. movimientos o partidos). Por lo tanto, para la presente investigación se trabaja con una muestra de 50 empresas evidenciando que en cada una de ellas exista un gestor de comunicación o responsable director de un área de comunicación o RRPP.

Una vez realizada la selección de las organizaciones objeto de estudio, se aplica la guía de entrevista a los responsables de la gestión de comunicación para identificar cuáles son sus competencias, determinar su campo de acción y conocer si cuentan o no con autonomía presupuestaria para desempeñar su gestión. Además, la interpretación de los textos obtenidos de las transcripciones de las entrevistas fue analizada a través del programa $\mathrm{NVivo}^{9}$. Se codificaron las variables de investigación que responden a los objetivos del estudio.

\footnotetext{
${ }^{8}$ Este proceso de evaluación, acreditación y re-categorización es producto del mandato constituyente No. 14 expedido por la Asamblea Nacional Constituyente el 22 de julio de 2008, y establece la obligación del "Consejo Nacional de Evaluación y Acreditación CONEA de entregar al Conesup y a la Función Legislativa, un informe técnico sobre el nivel de desempeño institucional de los establecimientos de Educación Superior, a fin de garantizar su calidad, propiciando su depuración y mejoramiento; según lo determinado en el artículo 91 de la LOES (Ley Orgánica de Educación Superior).

${ }^{9}$ NVivo es un software que se dirige a la investigación con métodos cualitativos y mixtos. Está diseñado para ayudarlo a organizar, analizar y encontrar perspectivas en datos no estructurados o cualitativos, como: entrevistas, respuestas de encuestas con preguntas abiertas, artículos, contenido de las redes sociales y la web.
} 
Imagen 1: Categorías comparadas por cantidad de referencias de codificación

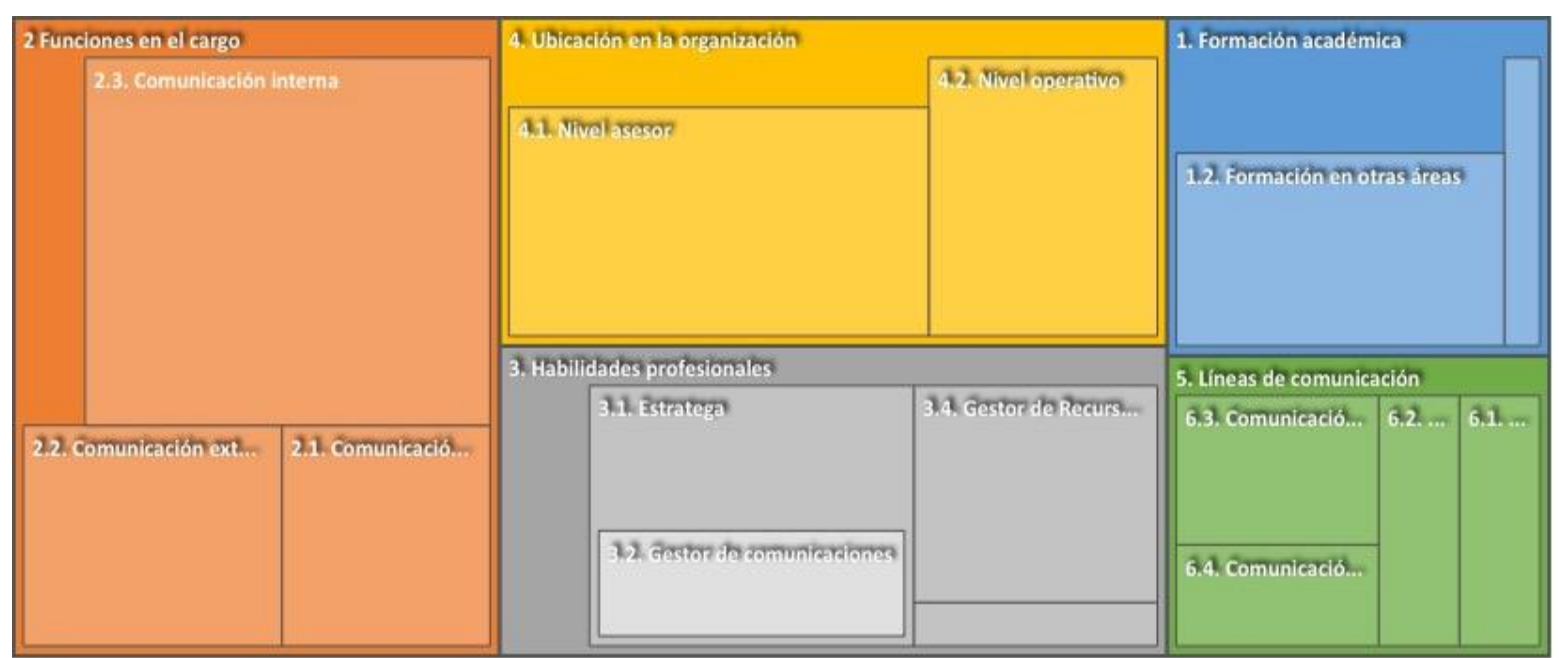

Fuente: Elaboración propia con datos de NVivo

\section{RESULTADOS}

Los resultados que a continuación se muestran corresponden al análisis realizado a 50 entrevistados, gestores de comunicación de las principales empresas del Ecuador de los sectores productivos: acuícola pesquero, alimentos, bebidas alcohólicas, comercial, comunicación, construcción, educativo, financiero, industrial, gubernamental, seguros, servicios, textil, vehículos y maquinarias y turístico. Los resultados están organizados en cuatro apartados: (1) formación académica, (2) funciones que desempeñan los Dircom en la organización, (3) habilidades profesionales, (4) nivel de gestión y decisorio.

\subsection{Formación académica}

Sin lugar a dudas el trabajo estratégico de la gestión de la comunicación exige una profesionalización forjada por la experiencia de los Dircom y por la formación que aportan las universidades. En la investigación se observa que el $42 \%$ de los gestores poseen entre 1 y 5 años de experiencia en la gestión de comunicación y un $18 \%$ del total de casos analizados tienen entre 6 y 14 años involucrados en el área. Este escenario se correlaciona perfectamente, pues la edad de los gestores 
de comunicación en un $68 \%$ está entre los 25 - 39 años, el siguiente grupo se ubica en un rango de 40 a 64 años.

Estamos por lo tanto frente a un grupo de responsables de comunicación de las empresas del país que recientemente finalizaron su carrera universitaria y que toman las riendas de los procesos de comunicación de las organizaciones; sin embargo, este grupo de profesionales avanza rápidamente en su formación, logrando alcanzar porcentajes cercanos entre los profesionales con tercer y cuarto nivel, tal es el caso que el $34 \%$ registra título de tercer nivel y un $28 \%$ de cuarto nivel. Existen también, algunos comunicadores que no cuentan con título universitario o poseen una titulación ajena a la comunicación, por ejemplo: contadores públicos autorizados (CPA) o ingenieros en administración de empresas, entre otros.

Gráfico 1: Titulación de los Dircom de Ecuador

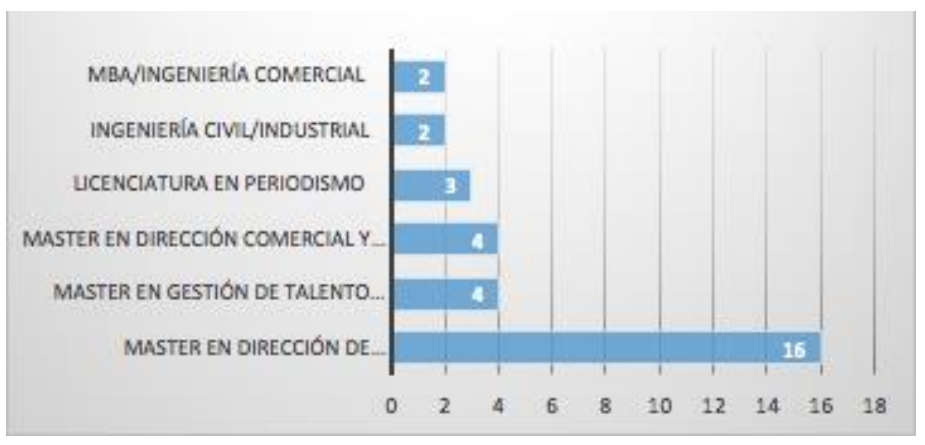

Fuente: Elaboración propia

Según datos reflejados por el Observatorio de la Comunicación Estratégica del Ecuador (2016), de una muestra de 130 encuestados, se observa un alto número de profesionales con títulos de licenciatura y magíster. Han pasado dos años, y aunque en una muestra menor (50 entrevistados), se observa que la tendencia se mantiene. A esta situación se debe sumar la falta de maestrías en el área. La plataforma Geoportal de la Secretaría de Educación Superior, Ciencia, Tecnología e Innovación (SENESCYT) a enero 2018, indica las universidades que ofertan carreras de pregrado en el área de la comunicación estratégica. Estas

REDMARKA IMARKA-Universidad de A Coruña - CIECID 
universidades, ubicadas en diversas zonas del país, dinamizan la presencia de gestores de comunicación en las organizaciones públicas y privadas. No hay una referencia exacta del número de titulados, pero según datos de la Tabla № 1 se puede observar que ninguna de las instituciones educativas cuenta con una formación sólida en la visión integral que tiene el Dircom.

Tabla 1: Oferta nacional de pregrado en comunicación estratégica

\begin{tabular}{|c|c|}
\hline $\begin{array}{l}\text { Universidad del Ecuador que ofertan } \\
\text { carreras de pregrado en comunicación } \\
\text { estratégica }\end{array}$ & Titulaciones del área. \\
\hline $\begin{array}{l}\text { Pontificia Universidad Católica del } \\
\text { Ecuador }\end{array}$ & $\begin{array}{l}\text { Ciencias de la comunicación mención } \\
\text { comunicación organizacional }\end{array}$ \\
\hline Universidad Casa Grande & $\begin{array}{l}\text { Comunicación con mención comunicación } \\
\text { organizacional }\end{array}$ \\
\hline Universidad de la Américas & Comunicación corporativa \\
\hline Universidad Internacional del Ecuador & Comunicación organizacional \\
\hline $\begin{array}{l}\text { Universidad Laica Eloy Alfaro de } \\
\text { Manabí }\end{array}$ & $\begin{array}{l}\text { Comunicación social con mención en relaciones } \\
\text { públicas y comunicación organizacional }\end{array}$ \\
\hline $\begin{array}{l}\text { Universidad } \quad \text { Particular } \\
\text { Especialidades Espíritu Santo }\end{array}$ & $\begin{array}{l}\text { Comunicación social en comunicación } \\
\text { organizacional y relaciones públicas }\end{array}$ \\
\hline Universidad Particular Internacional SEK & $\begin{array}{l}\text { Licenciatura en comunicación con énfasis en } \\
\text { comunicación empresarial }\end{array}$ \\
\hline Universidad Tecnológica SEK & $\begin{array}{l}\text { Licenciatura en comunicación énfasis relaciones } \\
\text { públicas }\end{array}$ \\
\hline Universidad Tecnológica Ecotec & Relaciones Públicas \\
\hline Universidad Tecnológica Equinoccial & $\begin{array}{ll}\text { Relaciones } & \text { Públicas } \\
\text { organizacional } & \end{array}$ \\
\hline Universidad Técnica Particular de Loja & Relaciones Públicas \\
\hline
\end{tabular}

Fuente: Geoportal, Senescyt. http://www.senescyt.gob.ec/visorgeografico/

El mismo portal revela que la oferta de maestrías en esta área es mínima. Solo las universidades Andina Simón Bolívar y Universidad de las Américas, ofertan las maestrías en Comunicación Estratégica y Dirección de Comunicación Empresarial

REDMARKA IMARKA-Universidad de A Coruña - CIECID

Año XI, Número 21, (2018), vol. 1 pp. 53-77 http://www.redmarka.net/ ISSN 1852-2300 
e Institucional, respectivamente; otras universidades, como la Casa Grande y Hemisferios, en su oferta de maestría en Comunicación se incluye componentes afines a la comunicación estratégica.

Es importante mencionar que el año 2016 en Consejo de Educación Superior CES del Ecuador expide el reglamento de armonización de la nomenclatura de títulos profesionales y grados académicos del Ecuador, en donde elimina las carreras afines a la comunicación estratégica, dejando esta área solo para las ofertas de maestría.

Estos antecedentes nos llevan a otro punto de análisis, que es los años que poseen en el mercado las organizaciones estudiadas y para las que trabajan los profesionales antes mencionados. Estas cuentan con una trayectoria de más de 10 años, tiempo elegido para el estudio ya que, además de coincidir con el periodo presidencial ininterrumpido más extenso que ha existido en el Ecuador, se considera que en este lapso ya han recuperado su inversión y están generando utilidades, dado que la Ley de Régimen Tributario Interno en el país contempla la exoneración de pago del Impuesto a la Renta durante cinco años para el desarrollo de inversiones nuevas y productivas.

Sin embargo, en la muestra seleccionada, esto no se traduce en la autonomía presupuestaria para los departamentos de comunicación, ya que solo 17 organizaciones, equivalentes al $34 \%$ de las empresas e instituciones seleccionadas, manifiestan contar con presupuestos exclusivos para la gestión de la comunicación, lo que puede atribuirse al panorama de desaceleración económica que ha existido en los últimos años.

Si bien entre los años 2006 y 2014 las 500 mayores empresas del país registraron incrementos en sus ingresos al pasar de US $\$ 35.985$ millones a US\$82.291 
millones, en los años siguientes se registraron descensos de US\$71.910 millones de dólares en 2015 y US $\$ 65.000$ en $2016^{10}$.

Cabe destacar que la presencia de multinacionales no siempre garantiza la introducción de los nuevos cambios organizacionales en cuanto a la dirección de comunicación se refiere, ya que de las 17 organizaciones en que los departamentos de comunicación cuentan con independencia presupuestaria, sólo dos corresponden a este tipo de empresas.

De la falta de disposición de recursos exclusivos para este departamento podría derivarse también que el Dircom no siempre cuente con un equipo de trabajo, de hecho de la muestra seleccionada, la cuarta parte de los gestores de la comunicación manifiestan que el personal a cargo que poseen está conformado por 1 a 5 integrantes, mientras en el $14 \%$ de los casos, los miembros del departamento están entre los 6 y 20. Existe un tercer grupo en que los equipos de trabajo sobrepasan las 50 personas, concretamente en 8 de los 50 casos investigados, pero esto se debe a que los encargados de la comunicación son los responsables de los departamentos de operaciones, recursos humanos o el mismo gerente general o gerente financiero.

\subsection{Gestión de comunicación}

Una de las principales características del Dircom es ser un profesional polivalente, con visión holística (Costa, 1999), con una figura de líder y con pensamiento estratega que organice la información que se genera desde la organización y lo que se dice de ella en función del quehacer productivo y/o servicios que ofrece, relación denominada por Costa (1999) como "Acción y Comunicación".

Todas las acciones y actuaciones de la empresa implican comunicación, a tal punto que la comunicación es más fuerte que la acción, pues tiene un mayor

\footnotetext{
${ }^{10}$ Esta evolución se obtiene de las cifras de ingresos anuales publicadas en las ediciones de 500 Mayores empresas del Ecuador de Revista Vistazo.
} 
impacto, se propaga y logra mantenerse en la memoria social. En la empresa toda decisión que se tome tiene que ser desarrollada y también comunicada. Se dice que actuar sin comunicar o también comunicar mal, es una de las peores decisiones que puede tomar, mucho más lo es, el comunicar y no actuar. La empresa debe ser transparente en su comunicación, de tal manera que sus públicos sientan realmente que se está compartiendo algo con ellos.

Las condiciones antes nombradas reflejan que el gestor de comunicación de las organizaciones tiene sobre sus hombros la visión integral del trabajo de la comunicación. En este sentido los Dircom de las empresas del Ecuador mencionan que el mayor porcentaje de su trabajo está dedicado a la comunicación integral, en algunos casos ejecutan la comunicación o en otros la destinan a agencias de comunicación. Sánchez y Pintado (2010:17) nos dice que la comunicación $360^{\circ}$ o comunicación integral tiene como objetivo gestionar de forma integrada y coherente las distintas vertientes de la comunicación organizacional o corporativa, como son: la imagen corporativa, las relaciones públicas, las campañas publicitarias y la web corporativa, entre los múltiples recursos que sirven para comunicar a los públicos.

Según los datos obtenidos se observa que, en los casos de empresas grandes, con más de 200 empleado, existen grupos de trabajo que apoyan la gestión de comunicación y es el director el encargado de vigilar la estrategia de comunicación.

"Nosotros elaboramos la comunicación interna y externa; sin embargo, tenemos una agencia de publicidad quien se encarga de hacer toda la implementación y todo lo que es el diseño de la comunicación que queremos implementar, es decir hacen las gráficas, el esquema y todo eso. También tenemos una agencia de relaciones públicas, en la que nos ayudan cuando hacemos 
algún lanzamiento de producto o algún evento en especial" (Álvarez, M., entrevista personal).

En el caso de las empresas analizadas los planes de comunicación externos los diseñan los comunicadores institucionales y las agencias contratadas, se otorga también a estas últimas la ejecución del plan; pero la comunicación interna queda en manos del equipo de comunicación y del departamento de recursos humanos.

La gráfica siguiente muestra en datos estadísticos la gestión de comunicación que realizan los Dircom.

Gráfico 2: Gestión de la comunicación del Dircom de Ecuador

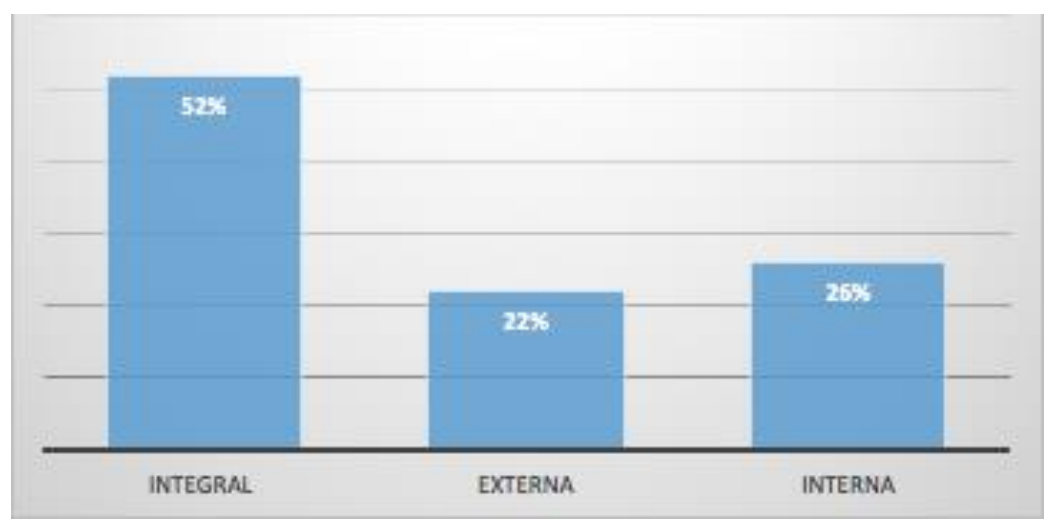

Fuente: Elaboración propia

La formación integral es otro de los elementos que aparece cuando se analiza el futuro del Dircom en las organizaciones ecuatorianas. Una formación que exige una visión que permita armonizar las relaciones de la organización con sus públicos internos y externos. Las empresas requieren profesionales responsables de la planificación estratégica y táctica, de la gestión de los valores intangibles, de la imagen pública y de la reputación institucional.

\subsection{Habilidades profesionales}

Enrique (2008: 342) asevera que el papel de los directores de comunicación ha cambiado en los últimos años. Ha pasado de ser un perfil totalmente operativo y

REDMARKA IMARKA-Universidad de A Coruña - CIECID

Año XI, Número 21, (2018), vol. 1 pp. 53-77

http://www.redmarka.net/ ISSN 1852-2300 
sin visión estratégica a ser de vital importancia y de reconocimiento de la alta esfera empresarial. En Ecuador, Yaguache y Elizalde (2010), Paladines y Yaguache (2015), Abendaño y Duque (2016) dan cuenta de un reconocimiento a la gestión que realiza el Dircom en las organizaciones; que nace desde una formación profesional sólida de tercer y cuarto nivel, hasta la visibilidad del trabajo que realizan las empresas.

El Observatorio de la Comunicación Estratégica (2015), indica de un estudio realizado en Ecuador, que entre las habilidades y conocimientos que debe tener el Dircom para su desempeño profesional, de acuerdo a criterios de académicos nacionales, es la destreza para aumentar su función estratégica como apoyo en la toma de decisiones. Esto es, sin lugar a dudas, la función clave del gestor de la comunicación, porque es aquella que le permitirá, posteriormente, hacer un balance del cumplimiento de objetivos que se señalan en la planificación estratégica.

Lo antes mencionado no está alejado de los resultados de las entrevistas realizadas en el octubre 2017. La opinión mayoritaria de los responsables de comunicación consultados indica que las empresas están reconociendo la importancia del Dircom como un gestor de reputación institucional, tiene mayores responsabilidades estratégicas y menos operativas.

Este hallazgo coincide con los resultados del Latin American Communication Monitor (2016 - 2017) donde 916 profesionales de la comunicación de 17 países, incluyendo a Ecuador consideraron que si bien los directores de los departamentos de comunicación dedican el $66 \%$ de su tiempo a actividades estratégicas y reflexivas como gestionar las actividades de comunicación y a los colaboradores; armonizar la comunicación entre la organización, el cliente y sus stakeholders, además de la orientación o coaching; la comunicación operacional les ocupa una tercera parte de su tiempo. 
A través de las respuestas obtenidas se evaluó cuál de las habilidades propuestas en la investigación es la que mayoritariamente ejecutan los Dircom de las empresas de Ecuador. Las variables propuestas fueron: estratega, gestor de comunicaciones, líder, gestor de RRHH.

La manifestación clara de los Dircom, según las actividades que ellos realizan, indica que la mayoría se considera estratega y, estratega y líder. Otros perfiles prefieren denominarse líder y gestor de comunicaciones; y algunos de ellos, por el trabajo de comunicación interna que ejecutan se consideran gestor de $\mathrm{RRHH}$ porque vigilan los procesos administrativos y de gestión operativa, aunque no trabajen precisamente en los despachos o direcciones de recursos humanos porque como ellos manifiestan:

Gráfico 3: Habilidades profesionales de los Dircom de Ecuador

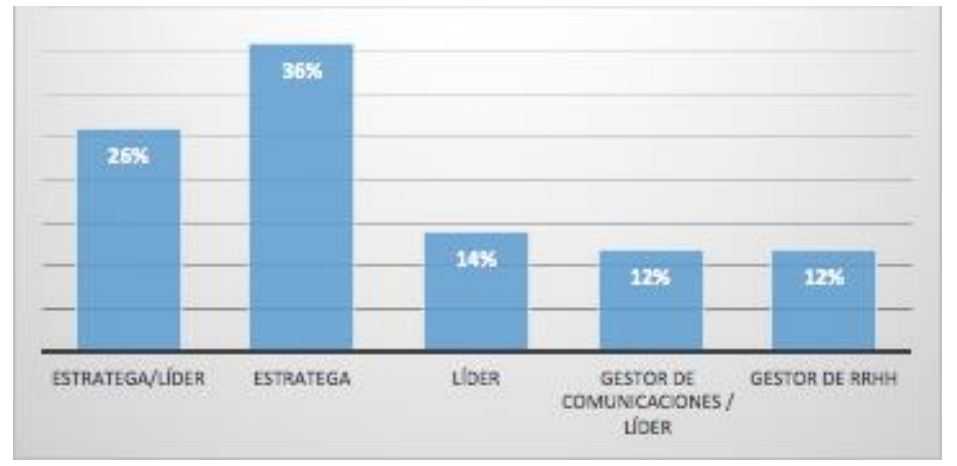

Fuente: Elaboración propia

"Estamos un poco satélite en el organigrama porque nosotros más salimos hacia al agricultor, vamos a campo todos los días, el equipo se dedica a eso principalmente" (Orrantia, R., entrevista personal).

"Para cualquier cambio interno se lo trabaja en conjunto con recursos humanos, ellos generan el contenido y yo hago la estrategia. La puesta en marcha lo hacemos en conjunto" (Álvarez, M., entrevista personal). 


\subsection{Ubicación en la organización}

En el año 2013, Burson-Marsteller en colaboración con Top Comunicación, editan el informe denominado "El Dircom del futuro y el futuro del Dircom", que recoge las sensaciones y reflexiones de 25 Dircom españoles acerca del rol que desempeñará la figura del director de comunicación y el propio departamento dentro de la estructura de las organizaciones. En este informe los entrevistados consideran que muchos directores de comunicación ya están integrados en los comités de dirección de las grandes empresas y que su trabajo se reporta al CEO o directamente a los altos mandos de la organización.

Los datos anteriores nos permiten determinar que en Ecuador la presencia del Dircom es cada vez más fuerte. Las variables antes analizadas favorecen esta premisa. La primera, la formación académica, en la que se evidencia que los responsables de comunicación de las organizaciones fortalecen sus conocimientos con estudios de cuarto nivel. Una segunda, la visión de ejercer una comunicación integral nos permite percibir el posicionamiento a favor en el organigrama de la empresa y por supuesto, como tercera, la habilidad profesional estratégica, declarada por los mismos entrevistados.

La relación de palabras, según el programa NVivo, permite visualizar articulaciones válidas sobre la importancia del Dircom como gestor y alineador de las acciones administrativas, productivas de comunicación de la organización.

Imagen 2: Relación de palabras con estratega

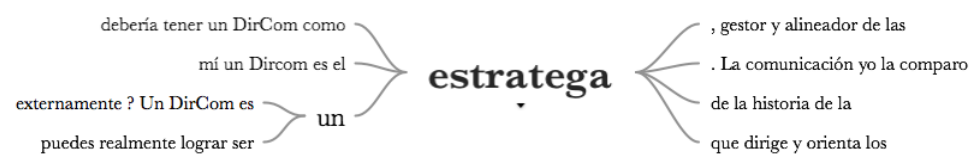

Fuente: Elaboración propia

En estadísticas, los resultados de las entrevistas analizadas por las respuestas de los Dircom indican un empoderamiento de las acciones de comunicación y de las

REDMARKA IMARKA-Universidad de A Coruña - CIECID 
líneas de relación y reporte directo con la comisión o consejo ejecutivo de las organizaciones. En el año 2015, el Observatorio de la Comunicación Estratégica del Ecuador, reporta en uno de sus informes que, de una muestra de 107 profesionales de la comunicación en actividad permanente, el $72.9 \%$ cree que aumentará la función estratégica del Dircom, como apoyo en la toma de decisiones de la alta gerencia; destacan, además, otras variables que evidencia el fortalecimiento de la comunicación estratégica.

Tabla 2: ¿Qué factores consolidarán la posición del director de comunicación en la organización?

\begin{tabular}{|c|c|}
\hline Opciones de respuesta & Porcentaje \\
\hline $\begin{array}{l}\text { Aumentará su función estratégica } \\
\text { como apoyo en la toma de decisiones } \\
\text { de la alta gerencia }\end{array}$ & $72,9 \%$ \\
\hline $\begin{array}{l}\text { Manejo de la microcomunicación } \\
\text { sobre la comunicación de masas } \\
\text { (grupos más segmentados) }\end{array}$ & $36,4 \%$ \\
\hline $\begin{array}{l}\text { Incorporar al modelo de gestión la } \\
\text { responsabilidad social y el desarrollo } \\
\text { sostenible }\end{array}$ & $35,5 \%$ \\
\hline $\begin{array}{l}\text { Conciliar la necesidad de abordar más } \\
\text { audiencias y canales con recursos } \\
\text { limitados }\end{array}$ & $28,0 \%$ \\
\hline Tendrá control de los nuevos medios & $21,5 \%$ \\
\hline Gestionar las crisis online & $21,5 \%$ \\
\hline 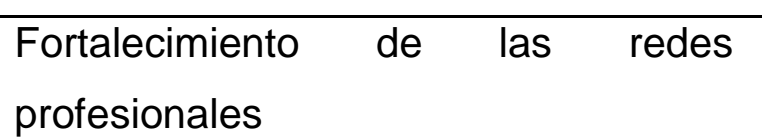 & $20,6 \%$ \\
\hline
\end{tabular}




\begin{tabular}{|ll|}
\hline $\begin{array}{l}\text { Concertar la velocidad y el volumen } \\
\text { del flujo de información }\end{array}$ & $17,8 \%$ \\
\hline Otro (especifique) & $2,8 \%$ \\
\hline
\end{tabular}

Fuente: Observatorio de la Comunicación Estratégica (2015)

Según los resultados de las entrevistas a los 50 Dircom de las empresas de Ecuador, se puede apreciar que la revalorización del Dircom se mantiene en un margen de crecimiento similar al del 2015. La visión holística de todo el fenómeno de la comunicación es importante para las organizaciones. Estamos, por lo tanto, frente a un perfil altamente necesario, capaz de colaborar en las decisiones estratégicas de la empresa y un gestor de información estratégico que coadyuve al crecimiento institucional y por ende al desarrollo del sector económico del Ecuador.

Gráfico 4: A quién reporta el Dircom de Ecuador

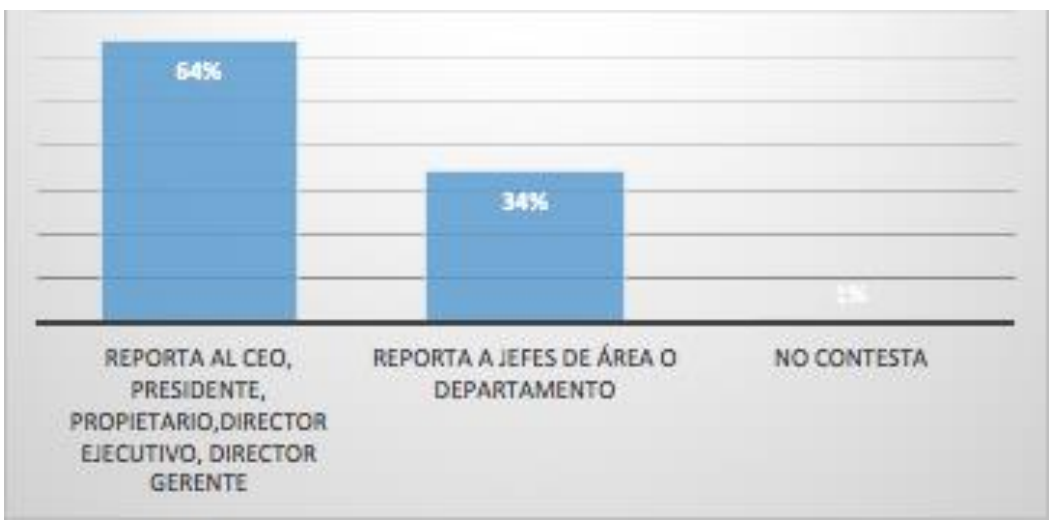

Fuente: Elaboración propia

"Mi cargo es debajo del presidente de la empresa en el área de recursos humanos el jefe de recursos humanos y se reporta al gerente general directamente" (Acevedo, A., entrevista personal).

"Somos más de dos mil quinientos colaboradores. Yo tengo el rango de jefe de comunicación. Somos alrededor de treinta y dos personas. La ventaja de mi posición es que yo respondo 
solamente para los dueños de la compañía, pero atiendo las necesidades de cada una de las áreas o proyectos que nazcan fuera de la misma" (Palacios, S., entrevista personal).

Frente a esta situación hay que reconocer que aún existe un número de gestores de comunicación que reportan a jefes de área o de departamento. En la investigación se identifican 15 entrevistados, cuatro de ellos, representantes de empresas familiares, en donde se suele encontrar ciertas dificultades de organización. Yaguache, J. et al. (2016: 953) al referirse a las empresas familiares, menciona que "hay una falta de políticas claras en temas como los puestos de trabajo de la familia en la empresa, la distribución de la riqueza, el sueldo de los miembros de la familia y cómo se van a mediar los conflictos".

Los resultados indican que no todos están en una dirección de comunicación, algunos de ellos son parte del área de recursos humanos o de ventas, pero las acciones que ejecutan son en el marco de la gestión de comunicación estratégica. La siguiente gráfica № 5 , indica la denominación del cargo que tienen los cincuenta entrevistados.

Gráfico 5: Denominación del cargo. Dircom Ecuador

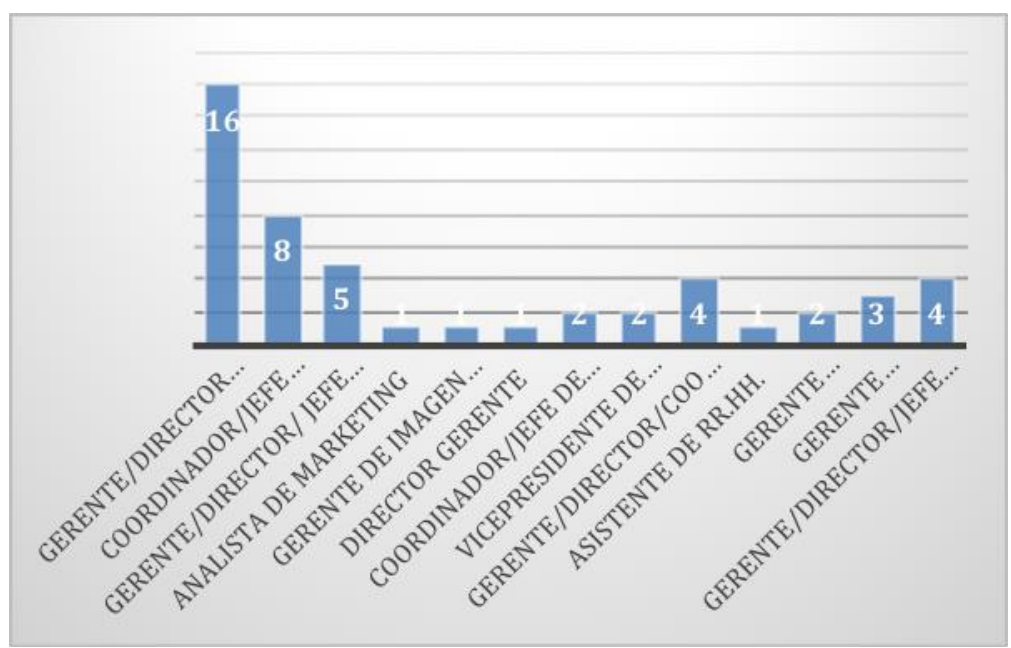

Fuente: Elaboración propia 


\section{DISCUSIONES Y CONCLUSIONES}

La investigación revela que los gestores de comunicación de las cincuenta empresas analizadas cuentan con un mínimo de 5 años de experiencia en la gestión de comunicación y un 18\% del total de casos analizados tienen entre 6 y 14 años de experiencia. La edad de los gestores de comunicación fluctúa entre los 25 - 39 años en un mayor porcentaje.

Las características anteriores inducen a determinar que los Dircom de Ecuador reconocen la importancia de su trabajo y el apoyo que brindan a las organizaciones por lo que se incrementa el número de profesionales, en actividad permanente, que cursan estudios de especialización en el área, exactamente el $34 \%$ registra título de tercer nivel y un $28 \%$ de cuarto nivel.

En cuanto a la gestión de comunicación que realizan se observa que su trabajo está enmarcado en la gestión de la comunicación integral, en algunos casos ellos mismo coordinan la comunicación desde sus departamentos y/o equipos de trabajo y en otros lo destinan a agencias de comunicación. Según las versiones de los entrevistados, en el caso de la comunicación externa se agencias contratadas y se otorga también a estas últimas la ejecución del plan; pero la comunicación interna queda en manos del equipo de comunicación y del departamento de recursos humanos.

En Ecuador, como en otros países, la posición del Dircom en el organigrama empresarial está mejor posicionada. Los resultados muestran que los Dircom ecuatorianos tiene mayores responsabilidades estratégicas y menos operativas. Según el trabajo y responsabilidades que tienen se denominan como estrategas de la comunicación porque vigilan todo el proceso interno y externo de proyección institucional.

A pesar del crecimiento y valorización del Dircom en la empresa ecuatoriana hay casos aún en los que no se logra que el empresario de cabida a la comunicación

REDMARKA IMARKA-Universidad de A Coruña - CIECID 
como eje estratégico de la organización. Existe un número de gestores de comunicación que reportan a jefes de área o de departamento en virtud de que no hay departamentos de comunicación o relaciones públicas debidamente conformados o a su vez, dependen de otras áreas como asuntos corporativos, marketing o recursos humanos.

También es importante destacar, que el $60 \%$ de los responsables de la comunicación son mujeres, esto coincide con el perfil demográfico de aquellos Dircom que respondieron a la encuesta de Latin American Monitor donde se identificó que el $66,6 \%$ son mujeres.

\section{REFERENCIAS BIBLIOGRÁFICAS}

Abendaño, M. \& Duque, V. (2016). El perfil del DirCom Interno en el Ecuador. De los medios y la comunicación de las organizaciones a las redes de valor. II XESCOM. Simposio Internacional.

Barbero, J. M. (2001). Reconfiguraciones comunicativas de lo público. Anàlisi: quaderns de comunicació i cultura, (26), 71-88.

Burns, Tom E. and Stalker, G.M., The Management of Innovation (1961). University of Illinois at Urbana-Champaign's Academy for Entrepreneurial Leadership Historical Research Reference in Entrepreneurship. Available at SSRN: https://ssrn.com/abstract=1496187

Costa, J. (1999): La comunicación en acción. Informe sobre la nueva cultura de la gestión, Barcelona, Paidós.

Costa, J. (2010). DirCom Estrategia de la complejidad, Nuevos paradigmas de la comunicación.

Dircom (2017) Manual de la Comunicación. Madrid, DIN Impresores. 
Elizalde, R., Yaguache, J. (2010). Diagnóstico del estado de las relaciones públicas en Ecuador desde el 2000 al 2010. Razón y Palabra [en linea] 2010, 15 (Noviembre-Sin mes) : [Fecha de consulta: 10 de marzo de 2018] Disponible en:<http://www.redalyc.org/articulo.oa?id=199516111035> ISSN 1605-4806

Galarza, F. Y. P., Quichimbo, J. Y., \& Benítez, V. A. (2016). Gestión de la comunicación estratégica en las organizaciones: enfoque ecuatoriano e internacional. Razón y palabra, (92), 49-32.

González, K. V., \& Ramírez, M. A. (2015). Historia y evolución de las Relaciones Públicas en Ecuador. María Isabel Punín Larrea, 33.

Hernández Carrera, R. M. (2014). La investigación cualitativa a través de entrevistas: su análisis mediante la teoría fundamentada. Cuestiones Pedagógicas, 23, 187-210.

Mut, M. (2006). El Director de Comunicación, perfil de una nueva figura. FISECEstrategias -Facultad de Ciencias Sociales de la Universidad Nacional de Lomas de Zamora, Año II, no 5, 3 - 23.

Observatorio de la Comunicación Estratégica del Ecuador. Universidad Técnica Particular de Loja.

Paladines, F., Yaguache, J., Altamirano, V. (2016). Gestión de la comunicación estratégica en las organizaciones: enfoque ecuatoriano e internacional. Revista Razón y Palabra, [S.I.], v. 20, n. 1_92, p. 1240-1271, mar. 2016. ISSN 1605-4806. Disponible en: $<$ http://www.revistarazonypalabra.org/index.php/ryp/article/view/369 $>$. Fecha de acceso: 10 mar. 2018

Punín Larrea, María Isabel, Los estudios de comunicación social/periodismo en el Ecuador. Una visión crítica al rol de la universidad y la academia. Razón y Palabra [en linea] 2012, 17 (Mayo-Julio): [Fecha de consulta: 5 de marzo de 2018] 
Disponible en:<http://www.redalyc.org/articulo.oa?id=199524411065> ISSN 16054806

Ritter, M. (2013). El valor del capital reputacional: por qué la opinión que el público tiene de su empresa es un activo estratégico. $R+P$ Buenos Aires.

Sánchez, J. \& Pintado, T. (2010). Estrategias de marketing para grupos sociales. España: ESIC Editorial.

Valarezo, K., Valdiviezo, C., Córdova, J. (2015). El rol estratégico de la Dirección de Comunicación en la Gestión de las Organizaciones. Revista Espacios. Vol. 36 (№ 13), Pág. 9.

Villafañe, J. (1999). La gestión profesional de la imagen corporativa. Ediciones Pirámide SA.

Yaguache Quichimbo, J. J., Rivera-Rogel, D. E., \& Campos Freire, F. (2016). Estrategias de las empresas periodísticas de Ecuador en el contexto de la crisis global de la prensa. Opción, 32(12). 